\title{
Crystal structure of (1,2-bi(4-pyridyl)ethane)(5-bromoisophthalato)- cobalt(II), $\mathrm{Co}\left(\mathrm{C}_{12} \mathrm{H}_{12} \mathrm{~N}_{2}\right)\left(\mathrm{C}_{8} \mathrm{H}_{3} \mathrm{O}_{4} \mathrm{Br}\right)$
}

\author{
Yun-Ping $\mathrm{Li}^{*} \cdot 1$ and Hong-Qiang $\mathrm{Ma}^{\mathrm{II}}$ \\ I Luoyang Normal University, Department of Chemistry, Luoyang, 471022 Henan, P. R. China \\ II Hong-Qi Middle School, Yanhu District, Yun Cheng, 044000 Shanxi, P. R. China
}

Received October 24, 2009, accepted and available on-line November 4, 2009; CCDC no. 1267/2821

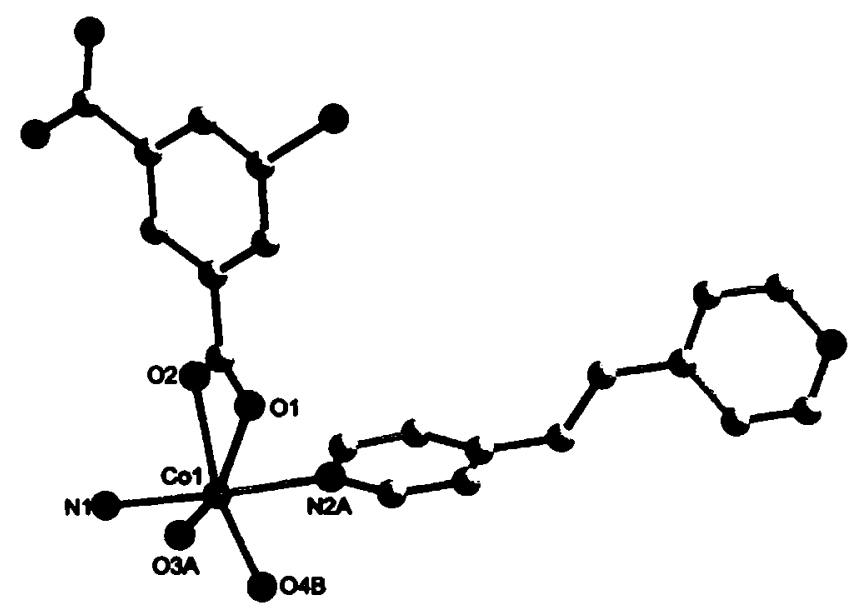

Abstract

$\mathrm{C}_{20} \mathrm{H}_{15} \mathrm{BrCoN}_{2} \mathrm{O}_{4}$, triclinic, $P \overline{1}$ (no. 2), $a=9.761(8) \AA$,

$b=10.086(8) \AA, c=11.202(9) \AA, \alpha=76.457(9)^{\circ}$,

$\beta=93.570(1)^{\circ}, \gamma=68.399(9)^{\circ}, V=983.3 \AA^{3}, Z=2$,

$R_{g t}(F)=0.038, w R_{\text {ref }}\left(F^{2}\right)=0.098, T=293 \mathrm{~K}$.

\section{Source of material}

A mixture of 5-bromoisophthalic acid $\left(\mathrm{H}_{2} 5\right.$-Br-ip, $0.1 \mathrm{mmol}$, $24.5 \mathrm{mg}$ ), 1,2-bi(4-pyridyl)ethane (bpa, $0.1 \mathrm{mmol}, 18.9 \mathrm{mg}$ ), $\mathrm{Co}(\mathrm{OAc})_{2} \cdot 4 \mathrm{H}_{2} \mathrm{O}(0.05 \mathrm{mmol}, 12.0 \mathrm{mg}), \mathrm{NaOH}(0.1 \mathrm{mmol}$, $4.0 \mathrm{mg})$ and $\mathrm{H}_{2} \mathrm{O}(15 \mathrm{~mL})$ was placed in a Teflon-lined stainless steel vessel, heated to $120^{\circ} \mathrm{C}$ for 3 days, and then cooled to room temperature over $24 \mathrm{~h}$. Pink block-shaped crystals of the title compound were obtained.

\section{Discussion}

The rational design and construction of coordination polymers with unique structural motifs and chemical or physical properties have attracted extensive interest in supramolecular and materials chemistry [1-8]. In the past decade, plenty of metal-organic hybrids exhibiting fascinating structures and various properties such as luminescence, magnetics, sorption, ion exchange, nonlinear optics and electricity have been synthesized by chemists. Among the various ligands, organic aromatic poly-carboxylates have been extensively employed in the preparation of such metalorganic compounds. In comparison to the 1,3-benzenedicarboxylic acid, 1,3,5-benzenetricarboxylic acid, and 1,2,4,5benzenetetracarboxylic acid ligands, 5-bromoisophthalic acid remains largely unexplored.
The asymmetric unit of the title structure contains one Co(II) cation, one bpa ligand and one 5-Br-ip anion. Each Co atom is octahedrally coordinated by two trans $\mathrm{N}$ atoms of two bpa ligands and four oxygen atoms from three 5-Br-ip ligands. The $\mathrm{Co}-\mathrm{N}$ bond lengths are both $2.163(3) \AA$ and the $\mathrm{Co}-O$ bond lengths are in the range of $2.011(3)-2.253(3) \AA$, respectively. X-ray structural analysis reveals that the title structure consists of a $2 D$ layer constructed from the linakge of $1 \mathrm{D}[\mathrm{Co}(\mathrm{bpa})]_{\mathrm{n}}$ chains via flexible bpa. Along the [110] direction, the Co atoms are bridged by the 5 Br-ip anions to generate $1 \mathrm{D}$ ribbon-like chains containing alternative arrangement of 8 - and 16 -membered rings. Two carboxylate groups bridge two Co(II) ions to form an 8membered ring and two 5-Br-ip ligands bridge two $\mathrm{Co}$ (II) ions to form a 16-membered ring. Within the 8-membered ring and 16membered ring, the Co $\cdots$. Co separations are 4.5 and $7.0 \AA$, respectively. The adjacent ribbon chains are interlinked by the flexible bpa ligands to form a 2D layer.

Table 1. Data collection and handling.

$\begin{array}{ll}\text { Crystal: } & \text { pink block, size } 0.06 \times 0.25 \times 0.35 \mathrm{~mm} \\ \text { Wavelength: } & \text { Mo } K_{a} \text { radiation }(0.71073 \AA) \\ \mu: & 11.55 \mathrm{~cm}^{-1} \\ \text { Diffractometer, scan mode: } & \text { Bruker SMART CCD, } \varphi / \omega \\ 2 \theta_{\text {max: }} & 51^{\circ} \\ N(h k d)_{\text {measured, }} N(h k l)_{\text {unique }}: & 6844,3558 \\ \text { Criterion for } I_{\text {obs }} N(h k l)_{\text {gt }}: & I_{\text {obs }}>2 \sigma\left(I_{\text {obs }}\right), 2605 \\ N(\text { param })_{\text {refined: }} & 253 \\ \text { Programs: } & \text { SHELXS-97 }[9], \text { SHELXL-97 [10], } \\ & \text { SHELXTL [11] }\end{array}$

Table 2. Atomic coordinates and displacement parameters (in $\AA^{2}$ ).

\begin{tabular}{llllll}
\hline Atom & Site & $x$ & \multicolumn{1}{l}{$y$} & $z$ & $U_{\text {iso }}$ \\
\hline H(2) & $2 i$ & 0.9186 & -0.0752 & 0.4102 & 0.034 \\
H(4) & $2 i$ & 0.8205 & -0.2279 & 0.1488 & 0.037 \\
H(6) & $2 i$ & 0.8184 & 0.1808 & 0.0826 & 0.042 \\
H(9) & $2 i$ & 0.8023 & 0.1531 & 0.5405 & 0.052 \\
H(10) & $2 i$ & 0.6164 & 0.1401 & 0.6918 & 0.054 \\
H(12) & $2 i$ & 0.5166 & 0.5580 & 0.6916 & 0.074 \\
H(13) & $2 i$ & 0.7026 & 0.5617 & 0.5397 & 0.070 \\
H(14A) & $2 i$ & 0.4271 & 0.2413 & 0.8419 & 0.050 \\
H(14B) & $2 i$ & 0.3358 & 0.4048 & 0.7953 & 0.050 \\
H(15A) & $2 i$ & 0.4673 & 0.4759 & 0.9101 & 0.063 \\
H(15B) & $2 i$ & 0.5596 & 0.3127 & 0.9561 & 0.063 \\
H(16) & $2 i$ & 0.0096 & 0.5335 & 1.1306 & 0.052 \\
H(17) & $2 i$ & 0.1816 & 0.5414 & 0.9657 & 0.057 \\
H(19) & $2 i$ & 0.4709 & 0.2006 & 1.1544 & 0.057 \\
H(20) & $2 i$ & 0.2903 & 0.2035 & 1.3145 & 0.053 \\
& & & & &
\end{tabular}


Table 3. Atomic coordinates and displacement parameters (in $\AA^{2}$ ).

\begin{tabular}{|c|c|c|c|c|c|c|c|c|c|c|}
\hline Atom & Site & $x$ & $y$ & $z$ & $U_{11}$ & $U_{22}$ & $U_{33}$ & $U_{12}$ & $U_{13}$ & $U_{23}$ \\
\hline $\operatorname{Co}(1)$ & $2 i$ & $0.95442(6)$ & $0.36598(5)$ & $0.38443(4)$ & $0.0401(3)$ & $0.0226(3)$ & $0.0241(3)$ & $-0.0199(2)$ & $0.0027(2)$ & $-0.0051(2)$ \\
\hline $\mathbf{B r}(1)$ & $2 i$ & $0.76664(6)$ & $0.00259(5)$ & $-0.06353(4)$ & $0.0923(4)$ & $0.0605(3)$ & $0.0370(3)$ & $-0.0345(3)$ & $-0.0267(3)$ & $-0.0017(2)$ \\
\hline$O(1)$ & $2 i$ & $0.8411(3)$ & $0.3168(3)$ & $0.2498(2)$ & $0.057(2)$ & $0.021(1)$ & $0.042(2)$ & $-0.019(1)$ & $-0.006(1)$ & $-0.003(1)$ \\
\hline$O(2)$ & $2 i$ & $0.9956(3)$ & $0.1482(3)$ & $0.3724(2)$ & $0.051(2)$ & $0.028(1)$ & $0.028(1)$ & $-0.025(1)$ & $-0.002(1)$ & $-0.005(1)$ \\
\hline$O(3)$ & $2 i$ & $0.9061(3)$ & $-0.3277(3)$ & $0.4888(2)$ & $0.053(2)$ & $0.030(1)$ & $0.027(2)$ & $-0.023(1)$ & $-0.009(1)$ & $0.000(1)$ \\
\hline$O(4)$ & $2 i$ & $0.8754(3)$ & $-0.4147(3)$ & $0.3349(2)$ & $0.049(2)$ & $0.025(1)$ & $0.032(1)$ & $-0.023(1)$ & $0.002(1)$ & $-0.007(1)$ \\
\hline$N(1)$ & $2 i$ & $0.7721(4)$ & $0.3591(3)$ & $0.5234(3)$ & $0.043(2)$ & $0.033(2)$ & $0.032(2)$ & $-0.024(2)$ & $0.007(2)$ & $-0.008(1)$ \\
\hline $\mathbf{N}(2)$ & $2 i$ & $0.1317(4)$ & $0.3681(3)$ & $1.2391(3)$ & $0.044(2)$ & $0.032(2)$ & $0.025(2)$ & $-0.023(2)$ & $0.005(1)$ & $-0.005(1)$ \\
\hline$C(1)$ & $2 i$ & $0.8753(4)$ & $0.0684(4)$ & $0.2523(3)$ & $0.040(2)$ & $0.025(2)$ & $0.031(2)$ & $-0.020(2)$ & $-0.000(2)$ & $-0.005(2)$ \\
\hline$C(2)$ & $2 i$ & $0.8919(4)$ & $-0.0617(4)$ & $0.3313(3)$ & $0.038(2)$ & $0.028(2)$ & $0.026(2)$ & $-0.021(2)$ & $0.001(2)$ & $-0.007(2)$ \\
\hline$C(3)$ & $2 i$ & $0.8689(4)$ & $-0.1736(4)$ & $0.2941(3)$ & $0.036(2)$ & $0.022(2)$ & $0.026(2)$ & $-0.017(2)$ & $0.001(2)$ & $-0.005(2)$ \\
\hline$C(4)$ & $2 i$ & $0.8328(4)$ & $-0.1529(4)$ & $0.1758(3)$ & $0.041(2)$ & $0.028(2)$ & $0.035(2)$ & $-0.021(2)$ & $-0.006(2)$ & $-0.008(2)$ \\
\hline$C(5)$ & $2 i$ & $0.8151(4)$ & $-0.0217(4)$ & $0.0978(3)$ & $0.047(3)$ & $0.035(2)$ & $0.028(2)$ & $-0.021(2)$ & $-0.009(2)$ & $-0.003(2)$ \\
\hline$C(6)$ & $2 i$ & $0.8336(4)$ & $0.0913(4)$ & $0.1351(3)$ & $0.053(3)$ & $0.026(2)$ & $0.031(2)$ & $-0.021(2)$ & $-0.008(2)$ & $0.000(2)$ \\
\hline$C(7)$ & $2 i$ & $0.9058(4)$ & $0.1864(4)$ & $0.2928(3)$ & $0.040(2)$ & $0.027(2)$ & $0.026(2)$ & $-0.021(2)$ & $0.006(2)$ & $-0.008(2)$ \\
\hline$C(8)$ & $2 i$ & $0.8868(4)$ & $-0.3161(4)$ & $0.3800(3)$ & $0.030(2)$ & $0.023(2)$ & $0.031(2)$ & $-0.015(2)$ & $0.000(2)$ & $-0.005(2)$ \\
\hline$C(9)$ & $2 i$ & $0.7441(5)$ & $0.2365(4)$ & $0.5712(4)$ & $0.056(3)$ & $0.037(2)$ & $0.036(2)$ & $-0.023(2)$ & $0.017(2)$ & $-0.008(2)$ \\
\hline$C(10)$ & $2 i$ & $0.6333(5)$ & $0.2276(5)$ & $0.6635(4)$ & $0.061(3)$ & $0.043(2)$ & $0.038(2)$ & $-0.033(2)$ & $0.013(2)$ & $-0.009(2)$ \\
\hline$C(11)$ & $2 i$ & $0.5481(4)$ & $0.3453(4)$ & $0.7139(3)$ & $0.037(2)$ & $0.048(2)$ & $0.027(2)$ & $-0.025(2)$ & $0.003(2)$ & $-0.006(2)$ \\
\hline$C(12)$ & $2 i$ & $0.5746(6)$ & $0.4730(5)$ & $0.6632(4)$ & $0.080(4)$ & $0.048(3)$ & $0.061(3)$ & $-0.036(3)$ & $0.037(3)$ & $-0.029(2)$ \\
\hline$C(13)$ & $2 i$ & $0.6860(6)$ & $0.4744(5)$ & $0.5710(4)$ & $0.082(4)$ & $0.044(3)$ & $0.058(3)$ & $-0.042(3)$ & $0.032(3)$ & $-0.022(2)$ \\
\hline$C(14)$ & $2 i$ & $0.4316(5)$ & $0.3387(5)$ & $0.8203(4)$ & $0.041(3)$ & $0.057(3)$ & $0.034(2)$ & $-0.029(2)$ & $0.007(2)$ & $-0.012(2)$ \\
\hline$C(15)$ & $2 i$ & $0.4633(5)$ & $0.3783(6)$ & $0.9320(4)$ & $0.049(3)$ & $0.086(4)$ & $0.040(3)$ & $-0.044(3)$ & $0.016(2)$ & $-0.023(2)$ \\
\hline$C(16)$ & $2 i$ & $0.1043(5)$ & $0.4650(5)$ & $1.1359(4)$ & $0.045(3)$ & $0.044(2)$ & $0.033(2)$ & $-0.014(2)$ & $0.008(2)$ & $-0.004(2)$ \\
\hline$C(17)$ & $2 i$ & $0.2073(5)$ & $0.4706(5)$ & $1.0360(4)$ & $0.059(3)$ & $0.049(3)$ & $0.030(2)$ & $-0.022(2)$ & $0.007(2)$ & $-0.001(2)$ \\
\hline$C(18)$ & $2 i$ & $0.3484(5)$ & $0.3713(5)$ & $1.0404(4)$ & $0.046(3)$ & $0.055(3)$ & $0.031(2)$ & $-0.036(2)$ & $0.008(2)$ & $-0.014(2)$ \\
\hline$C(19)$ & $2 i$ & $0.3772(5)$ & $0.2706(5)$ & $1.1469(4)$ & $0.039(3)$ & $0.059(3)$ & $0.041(3)$ & $-0.015(2)$ & $0.005(2)$ & $-0.013(2)$ \\
\hline$C(20)$ & $2 i$ & $0.2675(5)$ & $0.2728(5)$ & $1.2431(4)$ & $0.046(3)$ & $0.049(3)$ & $0.035(2)$ & $-0.019(2)$ & $0.001(2)$ & $-0.005(2)$ \\
\hline
\end{tabular}

\section{References}

1. Liu, C. S.; Yang, J.; Yue, Q.; Li, G. D.; Cao, J. J.; Li, G. H.; Chen, J. S.: Structures, photoluminescence, up-conversion, and magnetism of $2 \mathrm{D}$ and 3D rare-earth coordination polymers with multicarboxylate linkages. Inorg. Chem. 45 (2006) 2857-2865.

2. Yue, Q.; Yang, J.; Li, G. H.; Li, G. D.; Xu,W.; Chen, J. S.; Wang, S. N.: Three-dimensional 3d-4f heterometallic coordination polymers: synthesis, structures, and magnetic properties. Inorg. Chem. 44 (2005) 52415246

3. Gomez-Lor, B.; Gutierrez-Puebla, E.; Iglesias, M.; Monge, M. A.; RuizValero, C.; Snejko, N.: Novel 2D and 3D Indium Metal-Organic Frameworks: Topology and Catalytic Properties. Chem. Mater. 17 (2005) 25682573.

4. Wang, X. L.; Qin, C.; Wang, E. B.: Polythreading of Infinite 1D Chains into Different Structural Motifs: Two Poly(pseudo-rotaxane) Architectures Constructed by Concomitant Coordinative and Hydrogen Bonds. Cryst. Growth Des. 6 (2006) 439-443.

5. Chen, Z. L.; Liang, F. P.; Tang, X. F.; Chen,M. S.; Song, L. H.; Hu, R. X.: Syntheses and Structural Characterization of Two Nitrilotriacetate Cobalt Complexes. Z. Anorg. Allg. Chem. 631 (2006) 3092-3095.
6. Ma, A. Q.; Zhu, L. G.: Hydrothermal synthesis, conversion reaction, and crystal structure of 2-D 2-bromo-1,4-benzenedicarboxylate copper(II) complex with 2,2'-bipyridine. Transit. Metal Chem. 30 (2005) 869-872.

7. Shi, Q.; Cao, R.; Sun, D. F.;Hong, M. C.; Liang, Y. C.: Solvothermal syntheses and crystal structures of two metal coordination polymers with double-chain structures. Polyhedron 20 (2001) 3287-3293.

8. Shi, X.;Zhu, G. S.; Fang, Q. R.; Wu, G.; Tian, G.; Wang, R. W.; Zhang, D L.; Xue, M.; Qiu, S. L.: Novel Supramolecular Frameworks Self-Assembled from One-Dimensional Polymeric Coordination Chains. Eur. J. Inorg. Chem. (2004) 185-191.

9. Sheldrick, G. M.: SHELXS-97. Program for the Solution of Crystal Structures. University of Göttingen, Germany 1997.

10. Sheldrick, G. M.: SHELXI-97. Program for the Refinement of Crystal Structures. University of Göttingen, Germany 1997

11. Sheldrick, G. M.: SHELXTL. Structure Determination Software Suite Version 6.14. Bruker AXS, Madison, Wisconsin, USA 2000. 